\title{
The Vibrations of a Particle about a Position of Equilibrium.
}

By Bevan B. BAKer, Lecturer in Mathematics in the University of Edinburgh, and E. B. Ross, Professor of Mathematics in the Madras Christian College.

(Read 11th March 1921. Received 10th August 1921.)

1. In obtaining a solution of the differential equations corresponding to the motion of a particle about a position of equilibrium, it is usual to express the displacements in terms of a series of periodic terms, each sine or cosine having for its coefficient a series of powers of small quantities. Korteweg * has discussed the general form of such solutions, and, from the developments in series which he has obtained, has deduced certain features of interest. In particular, he has shown that, under certain circumstances, it is possible that certain vibrations of higher order, which are normally of small intensity compared with the principal vibrations, may acquire an abnormally large intensity. Considering the oscillations of a dynamical system having a number of degrees of freedom, and supposing $\frac{s_{1}}{2 \pi}, \frac{s_{2}}{2 \pi}, \ldots .$. to be the frequencies corresponding to infinitesimal oscillations in the different normal coordinates, Korteweg has shown that these cases of interest arise only when

$$
p_{1} s_{1}+p_{2} s_{2}+\ldots \ldots
$$

is zero or very small, where $p_{1}, p_{2}, \ldots$ are small integers, positive or negative; the most important cases occur when

$$
S \equiv\left|p_{1}\right|+\left|p_{2}\right|+\ldots \leqq 4 .
$$

The cases when $S \leqq 4$ have been discussed at some length by $B e t h, \dagger$ who uses, as an illustration of his dynamical system, the oscillations of a particle near the bottom of a bowl.

The case of two degrees of freedom, where $S=3$, i.e. $p_{1}=2$, $p_{z}=-1$, is the most important, and it is a particular form of this

* Korteweg: Archives Néerlandaises des Sciences exaotos et naturelles (2), 1, pp. 229-260, 1897.

†H. Beth: Archives Neerlandaises des Sciences exactes et naturelles (2), 15, pp. 246-283, 1910. 
case which is discussed in this paper. Under these conditions the radii of curvature at the bottom of the bowl are approximately in the ratio of $4: 1$.

The solution in periodic series has the disadvantage of becoming unmanageable for certain values of the frequencies, and it is difficult to determine whether this is due to actual divergence or whether the trouble arises from the presence of a part increasing steadily with the time (secular term). The particular problem discussed, which was suggested by Prof. E. T. Whittaker, has the advantage of being soluble not only in periodic series but also in terms of elliptic functions, and this second form of solution gives results where the series solution breaks down. This paper is chiefly concerned with a discussion of the conditions, obtained from the elliptic function solution, under which any valid solution of the problem exists. In a later paper it is hoped to apply the conditions so obtained to ascertain the cause of the divergence of the series solution.

2. Let $\phi_{1}$ and $\phi_{2}$ be the normal coordinates of the system, $\psi_{1}$ and $\psi_{2}$ the corrisponding momenta, and $\frac{s_{1}}{2 \pi}, \frac{s_{2}}{2 \pi}$, the frequencies of the principal vibrations. Apply a contact transformation, to another system of coordinates $p_{1}, p_{2}, q_{1}, q_{2}$, defined by

$$
\left.\begin{array}{l}
\phi_{1}=\sqrt{\frac{z q_{1}}{s_{1}}} \cdot \cos p_{1}, \phi_{2}=\sqrt{\frac{2 q_{2}}{s_{3}}} \cdot \cos p_{2}, \\
\psi_{1}=\sqrt{2 s_{1} q_{1}} \cdot \sin p_{1}, \psi_{2}=\sqrt{2 s_{2} q_{2}} \cdot \sin p_{2} .
\end{array}\right\}
$$

We assume Hamilton's function $H=T+V$, where $T$ and $V$ represent the kinetic and potential energies of the system, respectively, to be given by

$$
H=s_{1} q_{1}+s_{2} q_{2}+\alpha q_{1} q_{2}^{\frac{1}{2}} \cos \left(2 p_{1}-p_{2}\right),
$$

a being a certain constant.

This gives a somewhat artificial form to the kinetic and potential energies in terms of the original coordinates, viz.,

$$
\begin{aligned}
& T=\frac{1}{2} \psi_{1}^{2}+\frac{1}{2} \psi_{2}^{2}-\frac{\alpha}{2 \sqrt{2}} \frac{s_{2}^{\frac{1}{2}}}{s_{1}} \phi_{2} \psi_{1}^{2}+\frac{\alpha}{\sqrt{2 .} s_{2}^{\frac{1}{2}}} \phi_{1} \psi_{1} \psi_{2}, \\
& V=\frac{1}{2} s_{1}^{2} \phi_{1}^{2}+\frac{1}{2} s_{2}^{2} \phi_{2}^{2}+\frac{\alpha}{2 \sqrt{2}} s_{1} s_{2}^{1} \phi_{1}^{2} \phi_{2 .} .
\end{aligned}
$$


The equations of motion of the system are

$$
\left.\begin{array}{l}
\frac{d q_{1}}{d t}=\frac{\partial H}{\partial p_{1}}, \frac{d q_{2}}{d t}=\frac{\partial H}{\partial p_{2}}, \\
\frac{d p_{1}}{d t}=-\frac{\partial H}{\partial q_{1}}, \frac{d p_{2}}{d t}=-\frac{\partial H}{\partial q_{2}} ;
\end{array}\right\}
$$

which, on substituting for $H$ from (2), give

$$
\begin{aligned}
& \frac{d q_{1}}{d t}=-2 \alpha q_{1} q_{2}^{\frac{1}{2}} \sin \left(2 p_{1}-p_{2}\right), \\
& \frac{d q_{2}}{d t}=\alpha q_{1} q_{2}^{\frac{1}{2}} \sin \left(2 p_{1}-p_{2}\right), \\
& \frac{d p_{1}}{d t}=-s_{1}-\alpha q_{\frac{1}{3}} \cos \left(2 p_{1}-p_{2}\right), \\
& \frac{d p_{2}}{d t}=-s_{2}-\frac{1}{2} \alpha \frac{q_{1}}{q_{2}^{\frac{l}{2}}} \cos \left(2 p_{1}-p_{2}\right) .
\end{aligned}
$$

Since the energy, $H$, of the system is constant, we have $H=s_{1} q_{1}+s_{2} q_{2}+\alpha q_{1} q_{2}^{\ddagger} \cos \left(2 p_{1}-p_{2}\right)=$ constant $=h$ (say) Also, from equations (4),

$$
\begin{aligned}
& \frac{d q_{1}}{d t}+2 \cdot \frac{d q_{2}}{d t}=0 ; \\
& \therefore q_{1}+2 q_{2}=\text { constant }=c \text { (say) } \ldots \ldots \ldots \ldots \ldots
\end{aligned}
$$

Having therefore two integrals, (5) and $(6)$, we can integrate the whole system.

3. Solution in Series.

We proceed first to obtain a solution in the form of infinite series.

From equations (5) and (6) we find

$$
\left.\begin{array}{l}
q_{1}=k_{1}^{2}-\frac{2 \alpha q_{1} q_{2}^{3}}{\left(2 s_{1}-s_{2}\right)} \cos \left(2 p_{1}-p_{2}\right), \\
q_{2}=k_{2}^{2}+\frac{\alpha q_{1} q_{2}^{2}}{\left(2 s_{1}-s_{2}\right)} \cos \left(2 p_{1}-p_{2}\right),
\end{array}\right\}
$$

where $k_{1}$ and $k_{2}$ are constants. 
Solving equations (7) by successive approximations, and putting $\phi=2 p_{1}-p_{2}$, we obtain

$$
\begin{aligned}
& q_{1}=k_{1}^{2}\left[1-\frac{\alpha^{2}}{2} \frac{\left(k_{1}^{2}-4 k_{2}^{2}\right)}{\left(2 s_{1}-s_{2}\right)^{2}}+\frac{3}{2} \frac{\alpha^{4}}{\left(2 s_{1}-s_{2}\right)^{4}}\left(k_{1}^{4}-6 k_{1}^{2} k_{2}^{2}+4 k_{2}^{4}\right)\right. \\
& -\frac{2 \alpha k_{2}}{\left(2 s_{1}-s_{2}\right)} \cos \phi-\frac{3}{16} \frac{\alpha^{3}}{\left(2 s_{1}-s_{2}\right)^{3}} \frac{\left(k_{1}^{4}-24 k_{1}^{2} k_{2}^{2}+32 k_{2}^{4}\right)}{k_{9}} \cos \phi \\
& -\frac{\alpha^{2}}{2} \frac{\left(k_{1}^{2}-4 k_{2}^{2}\right)}{\left(2 s_{1}-s_{2}\right)^{2}} \cos 2 \phi+\frac{2 \alpha^{4}}{\left(2 s_{1}-s_{2}\right)^{4}}\left(k_{1}^{4}-6 k_{1}^{2} k_{2}^{2}+4 k_{2}^{4}\right) \cos 2 \phi \\
& -\frac{1}{16} \frac{\alpha^{3}}{\left(2 s_{1}-s_{2}\right)^{3}} \frac{\left(k_{1}^{4}-24 k_{1}^{2} k_{2}^{2}+32 k_{2}^{ \pm}\right)}{k_{2}} \cos 3 \phi \\
& \left.+\frac{1}{2} \frac{\alpha^{4}}{\left(28_{1}-s_{2}\right)^{4}}\left(k_{1}^{4}-6 k_{1}^{2} k_{2}^{2}+4 k_{2}^{4}\right) \cos 4 \phi\right] \text {, } \\
& q_{2}=k_{2}^{2}\left[1+\frac{1}{4} \frac{\alpha^{2}}{\left(2 s_{1}-s_{2}\right)^{2}} \frac{k_{1}^{2}}{k_{2}^{2}}\left(k_{1}^{2}-4 k_{2}^{2}\right)-\frac{3}{4} \frac{\alpha^{4}}{\left(2 s_{1}-s_{2}\right)^{4}} \frac{k_{1}^{2}}{k_{2}^{2}}\left(k_{1}^{4}-6 k_{1}^{2} k_{2}^{2}+4 k_{2}^{4}\right)\right. \\
& +\frac{\alpha}{\left(2 s_{1}-s_{2}\right)} \frac{k_{1}^{2}}{k_{2}} \cos \phi+\frac{3}{32} \frac{\alpha^{3}}{\left(2 s_{1}-s_{2}\right)^{3}} \frac{k_{1}^{2}}{k_{2}^{3}}\left(k_{1}^{4}-24 k_{1}^{2} k_{2}^{2}+32 k_{2}^{4}\right) \cos \phi \\
& +\frac{1}{4} \frac{\alpha^{3}}{\left(2 s_{1}-B_{2}\right)^{2}} \frac{k_{1}^{2}}{k_{2}^{2}}\left(k_{1}^{2}-4 k_{2}^{2}\right) \cos 2 \phi \\
& -\frac{\alpha^{4}}{\left(2 s_{1}-s_{2}\right)^{4}} \frac{k_{1}^{2}}{k_{2}^{2}}\left(k_{1}^{4}-6 k_{1}^{2} k_{2}^{2}+4 k_{2}^{4}\right) \cos 2 \phi \\
& +\frac{1}{32} \frac{\alpha^{3}}{\left(2 g_{1}-8_{2}\right)^{3}} \frac{k_{1}^{2}}{k_{2}^{3}}\left(k_{1}^{4}-24 k_{1}^{2} k_{2}^{2}+32 k_{2}^{4}\right) \cos 3 \phi \\
& \left.-\frac{1}{4} \frac{\alpha^{4}}{\left(2 s_{1}-s_{2}\right)^{4}} \frac{k_{1}^{2}}{k_{2}^{2}}\left(k_{1}^{4}-6 k_{1}^{2} k_{2}^{2}+4 k_{2}^{4}\right) \cos 4 \phi\right],
\end{aligned}
$$

correct to the sixth power of the small quantities $k_{1}$ and $k_{2}$.

Now, from the general theory, $q_{1} d p_{1}+q_{2} d p_{2}$ is a perfect differential, $d W$ (say);

$$
\begin{aligned}
& \therefore q_{1}=\frac{\partial W}{\partial p_{1}}, q_{2}=\frac{\partial W}{\partial p_{2}} \\
& \therefore W=\left\{k_{1}^{2}-\frac{\alpha^{2}}{2} \frac{k_{1}^{2}\left(k_{1}^{2}-4 k_{2}^{2}\right)}{\left(2 s_{1}-8_{2}\right)^{2}}+\frac{3}{2} \frac{\alpha^{4}}{\left(2 s_{1}-8_{2}\right)^{4}} k_{1}^{2}\left(k_{1}^{4}-6 k_{1}^{2} k_{2}^{2}+4 k_{2}^{4}\right)\right\} p_{1}
\end{aligned}
$$


$+\left\{k_{2}^{2}+\frac{1}{4} \frac{\alpha^{2}}{\left(2 \varepsilon_{1}-s_{2}\right)^{2}} k_{1}^{2}\left(k_{1}^{2}-4 k_{3}^{2}\right)-\frac{3}{4} \frac{\alpha^{4}}{\left(2 s_{1}-s_{2}\right)^{4}} k_{1}^{2}\left(k_{1}^{4}-6 k_{1}^{2} k_{2}^{2}+4 k_{2}^{4}\right)\right\} p_{2}$ $-\frac{\alpha}{\left(2 s-s_{2}\right)} k_{1}^{2} k_{2} \sin \phi$

$-\frac{3}{32} \frac{\alpha^{3}}{\left(2 s-s_{2}\right)^{3}} \frac{k_{1}^{2}}{k_{2}}\left(k_{1}^{4}-24 k_{1}^{2} k_{2}^{2}+32 k_{2}^{4}\right) \sin \phi$

$-\frac{1}{8} \frac{\alpha_{2}}{\left(2 s_{1}-s_{2}\right)^{2}} k_{1}^{2}\left(k_{1}^{2}-4 k_{2}^{2}\right) \sin 2 \phi+\frac{1}{2} \frac{\alpha^{4}}{\left(2 s_{1}-s_{2}\right)^{4}} k_{1}^{2}\left(k_{1}^{4}-6 k_{1}^{2} k_{2}^{2}+4 k_{2}^{4}\right) \sin 2 \phi$ $-\frac{1}{96} \frac{\alpha^{3}}{\left(2 s_{1}-s_{2}\right)^{3}} \frac{k_{1}^{2}}{k_{2}}\left(k_{1}^{4}-24 k_{1}^{2} k_{2}^{2}+32 k_{2}^{4}\right) \sin 3 \phi$

$$
+\frac{1}{16} \frac{\alpha^{4}}{\left(2 s_{1}-s_{2}\right)^{4}} k_{1}^{2}\left(k_{1}^{4}-6 k_{1}^{2} k_{2}^{2}+4 k_{2}^{4}\right) \sin 4 \phi .
$$

Substituting $a_{1}$ and $a_{2}$ for $k_{1}$ and $k_{2}$, where $a_{1}$ and $a_{2}$ are given by

$$
\begin{aligned}
& a_{1}=k_{1}^{2}-\frac{\alpha^{2}}{2} \frac{k_{1}^{2}\left(k_{1}^{2}-4 k_{2}^{2}\right)}{\left(2 s_{1}-s_{2}\right)^{2}}+\frac{3}{2} \frac{\alpha^{4}}{\left(2 s_{1}-s_{2}\right)^{4}} k_{1}^{2}\left(k_{1}^{4}-6 k_{1}^{2} k_{2}^{2}+4 k_{2}^{4}\right), \\
& a_{2}=k_{2}^{2}+\frac{1}{4} \frac{\alpha^{2}}{\left(2 s_{1}-s_{2}\right)^{2}} k_{1}^{2}\left(k_{1}^{2}-4 k_{2}^{2}\right)-\frac{3}{4} \frac{\alpha^{4}}{\left(2 s_{1}-s_{2}\right)^{4}} k_{1}^{2}\left(k_{1}^{4}-6 k_{1}^{2} k_{2}^{2}+4 k_{2}^{4}\right),
\end{aligned}
$$

we obtain

$$
\begin{aligned}
W=a_{1} p_{1}+a_{2} p_{2}-\left\{\frac{a}{\left(2 s_{1}-s_{2}\right)} a_{1} a^{\frac{1}{2}}-\frac{1}{32} \frac{a^{3}}{\left(2 s_{1}-s_{2}\right)^{3}} \times\right. \\
\left.\quad\left(\frac{a_{1}^{3}}{a_{2}^{\frac{1}{2}}}+40 a_{1}^{2} a_{2}^{\frac{1}{2}}-32 a_{1} a_{2}^{3}\right)\right\} \sin \phi \\
-\left\{\frac{1}{8} \frac{\alpha^{2}}{\left(2 s_{1}-s_{2}\right)^{2}} a_{1}\left(a_{1}-4 a_{2}\right)\right. \\
\left.\quad-\frac{1}{4} \frac{\alpha^{4}}{\left(2 s_{1}-s_{1}\right)^{4}}\left(a_{1}^{3}-7 a_{1}^{2} a_{2}+4 a_{1} a_{2}^{3}\right)\right\} \sin 2 \phi \\
-\frac{1}{96} \frac{\alpha^{3}}{\left(2 s_{1}-s_{2}\right)^{3}}\left(\frac{a_{1}^{3}}{a_{2}^{3}}-24 a_{1}^{2} a_{2}^{\frac{1}{2}}+32 a_{1} a_{2}\right) \sin 3 \phi \\
+\frac{1}{16} \frac{\alpha^{4}}{\left(2 s_{1}-s_{2}\right)^{4}}\left(a_{1}^{3}-6 a_{1}^{2} a_{2}+4 a_{1} a_{2}^{2}\right) \sin 4 \phi .
\end{aligned}
$$

Now let $\beta_{1}=\frac{\partial W}{\partial a_{1}}$ and $\beta_{2}=\frac{\partial W}{\partial a_{2}}$;

then, from the general theory, the other two integrals required to complete the solution are

$$
\beta_{1}=\epsilon_{1}-t \cdot \frac{\partial H}{\partial a_{1}} \text { and } \beta_{2}=\epsilon_{2}-t \cdot \frac{\partial H}{\partial a_{2}},
$$

where $\epsilon_{1}$ and $\epsilon_{2}$ are arbitrary constants. 
From equations (8) and (9) we obtain values for $q_{1}$ and $q_{2}$, and, substituting these in equation (5), we get

$$
\begin{aligned}
H=s_{1} a_{1}+s_{2} a_{2}+ & \frac{1}{4} a_{1}\left(a_{1}-4 a_{2}\right) \frac{\alpha^{2}}{\left(2 s_{1}-s_{2}\right)} \\
& -\frac{1}{4} a_{1}\left(a_{1}^{2}-8 a_{1} a_{2}+4 a_{2}^{2}\right) \frac{\alpha^{4}}{\left(2 s_{1}-s_{2}\right)^{4}}
\end{aligned}
$$

From equations (11) and (12) we obtain values for $\beta_{1}$ and $\beta_{9}$ viz. :-

$$
\begin{aligned}
& \beta_{1}=\epsilon_{1}-t\left\{s_{1}+\right. \frac{1}{2} \frac{\alpha^{2}}{\left(2 s_{1}-s_{2}\right)}\left(a_{1}-2 a_{2}\right) \\
&\left.-\frac{1}{4} \frac{\alpha^{4}}{\left(2 s_{1}-s_{2}\right)^{3}}\left(3 a_{1}^{2}-16 a_{1} a_{2}+4 a_{2}^{2}\right)\right\}, \\
& \beta_{2}=\epsilon_{2}-t\left\{s_{2}-\frac{\alpha^{2}}{\left(2 s_{1}-s_{2}\right)} a_{1}+2 \frac{\alpha^{4}}{\left(2 s_{1}-s_{2}\right)^{3}} a_{1}\left(a_{1}-a_{2}\right)\right\}
\end{aligned}
$$

Writing now $M$ for $\left(2 \beta_{1}-\beta_{2}\right)$, we have

$$
\begin{aligned}
M=2 \epsilon_{1}-\epsilon_{2}-t\left\{\left(2 s_{1}-s_{2}\right)+2\left(a_{1}-a_{2}\right) \frac{\alpha^{2}}{\left(2 s_{1}-s_{2}\right)}\right. \\
\left.-\frac{1}{2}\left(7 a_{1}^{2}-20 a_{1} a_{2}+4 a_{2}^{2}\right) \frac{\alpha^{4}}{\left(2 s_{1}-s_{2}\right)^{3}}\right\}
\end{aligned}
$$

Also from (10) we obtain values for $p_{1}$ and $p_{2}$, giving

$$
\begin{aligned}
\phi \equiv 2 p_{1}-p_{2}=M & -\left\{\frac{1}{2} \frac{a}{\left(2 s_{1}-s_{2}\right)} \frac{\left(a_{1}-4 a_{2}\right)}{a_{\frac{1}{2}}^{\frac{1}{2}}}\right. \\
& \left.+\frac{1}{64} \frac{a^{3}}{\left(2 s_{1}-s_{2}\right)^{3}}\left(\frac{a_{1}^{3}}{a_{2}^{\frac{3}{2}}}-\frac{28 a_{1}^{2}}{a_{2}^{\frac{1}{2}}}+416 a_{1} a_{2}^{\frac{3}{3}}-128 a_{2}^{\frac{3}{3}}\right)\right\} \sin \phi \\
+ & \left\{\frac{a^{2}}{\left(2 s_{1}-s_{2}\right)^{2}}\left(a_{1}-a_{2}\right)-\frac{1}{4} \frac{a^{4}}{\left(2 s_{1}-s_{2}\right)^{4}}\left(13 a_{1}^{2}-36 a_{1} a_{2}+8 a_{2}^{2}\right)\right\} \sin 2 \phi \\
+ & \frac{1}{192} \frac{a^{3}}{\left(2 s_{1}-s_{2}\right)^{3}}\left(\frac{a_{1}^{3}}{a_{2}^{\frac{3}{3}}}+\frac{36 a_{1}^{2}}{a_{2}^{\frac{1}{2}}}-288 a_{1} a_{2}^{\frac{1}{2}}+128 a_{2}^{\frac{3}{2}}\right) \sin 3 \phi \\
- & \frac{1}{8} \frac{a^{4}}{\left(2 s_{1}-s_{2}\right)^{4}}\left(6 a_{1}^{2}-16 a_{1} a_{2}+4 a_{2}^{2}\right) \sin 4 \phi
\end{aligned}
$$




$$
\begin{aligned}
& =M-\left\{\frac{1}{2} \frac{\alpha}{\left(2 s_{1}-s_{2}\right)} \frac{\left(a_{1}-4 a_{2}\right)}{a_{2}^{\frac{1}{2}}}\right. \\
& \left.-\frac{1}{2} \frac{a^{3}}{\left(2 s_{1}-8_{2}\right)^{3}}\left(\frac{a_{1}^{2}}{a_{2}^{\frac{3}{2}}}-14 a_{1} a_{\frac{1}{2}}+4 a_{\frac{3}{2}}^{\frac{3}{2}}\right)\right\} \sin M \\
& +\left\{\frac{1}{8} \frac{\alpha^{2}}{\left(2 s_{1}-s_{2}\right)^{2}} \frac{\left(a_{1}^{2}+8 a_{2}^{2}\right)}{a_{2}}\right. \\
& \left.-\frac{1}{64} \frac{a^{4}}{\left(2 s_{1}-s_{2}\right)^{4}}\left(\frac{a_{1}^{4}}{a_{2}^{2}}+\frac{8 a_{1}^{3}}{a_{2}}-40 a_{1}^{2}+256 a_{1} a_{2}\right)\right\} \sin 2 M \\
& -\frac{1}{48} \frac{a^{3}}{\left(2 s_{1}-s_{2}\right)^{3}}\left(\frac{2 a_{1}^{3}}{a_{2}^{\frac{3}{2}}}-\frac{18 a_{1}^{2}}{a_{2}^{\frac{7}{3}}}+63 a_{1} a_{2}^{\frac{1}{2}}-104 a_{2}^{\frac{3}{2}}\right) \sin 3 M \\
& -\frac{1}{384} \frac{a^{4}}{\left(2 s_{1}-s_{2}\right)^{4}}\left(\frac{3 a_{1}^{4}}{a_{2}^{2}}-\frac{72 a_{1}^{3}}{a_{2}}+216 a_{1}^{2}+424 a_{1} a_{2}+960 a_{2}^{2}\right) \sin 4 M
\end{aligned}
$$

4. On substituting from (15) in the expressions for $q_{1}, q_{2}, p_{1}$, and $p_{2}$, we obtain finally the results

$$
\begin{aligned}
& q_{1}=a_{1}-2 a_{1} a_{2}^{\frac{1}{2}} \frac{\alpha}{\left(2 s_{1}-s_{2}\right)} \cos M-\frac{1}{2} a_{1}\left(a_{1}-4 a_{2}\right) \frac{\alpha^{2}}{\left(2 s_{1}-s_{2}\right)^{2}} \\
& +4 a_{1} a_{2}^{\ddagger}\left(a_{1}-a_{2}\right) \frac{a^{3}}{\left(2 s_{1}-s_{2}\right)^{3}} \cos M+\left\{\frac{3}{2} a_{1}\left(a_{1}^{2}-8 a_{1} a_{2}+4 a_{2}^{2}\right)\right. \\
& \left.+2 a_{1}^{2} a_{2} \cos 2 M\right\} \frac{a^{4}}{\left(2 s_{1}-s_{2}\right)^{4}}, \\
& q_{2}=a_{2}+a_{1} a_{2}^{\frac{3}{3}} \frac{\alpha}{\left(2 s_{1}-s_{2}\right)} \cos M+\frac{1}{4} a_{1}\left(a_{1}-4 a_{2}\right) \frac{\alpha^{2}}{\left(2 s_{1}-s_{2}\right)^{2}} \\
& -2 a_{1} a_{2}^{\frac{3}{2}}\left(a_{1}-a_{2}\right) \frac{\alpha^{3}}{\left(2 s_{1}-s_{2}\right)^{3}} \cos M \\
& -\left\{\frac{3}{4} a_{1}\left(a_{1}^{2}-8 a_{1} a_{2}+4 a_{2}^{2}\right)+4 a_{1}^{2} a_{2} \cos 2 M\right\} \frac{\alpha^{4}}{\left(2 s_{1}-s_{2}\right)^{4}}, \\
& p_{1}=\beta_{1}+a_{2}^{\frac{1}{2}} \frac{\alpha}{\left(2 s_{1}-s_{2}\right)} \sin M+\frac{1}{2} a_{2} \frac{\alpha^{2}}{\left(2 s_{1}-s_{2}\right)^{2}} \sin 2 M \\
& -\left\{\left(\frac{5}{2} a_{1} a_{2}^{\frac{1}{3}}-a_{2}^{\frac{3}{3}}\right) \sin M-\frac{1}{3} a_{2}^{\frac{3}{3}} \sin 3 M\right\} \frac{\alpha^{3}}{\left(2 s_{1}-s_{2}\right)^{3}} \\
& -\left\{\left(\frac{5}{2} a_{1} a_{2}-a_{2}^{2}\right) \sin 2 M-\frac{1}{4} a_{2}^{2} \sin 4 M\right\} \frac{\alpha^{4}}{\left(2 s_{1}-s_{2}\right)^{4}}, \\
& p_{2}=\beta_{2}+\frac{1}{2} \frac{a_{1}}{a_{2}^{\frac{1}{2}}} \frac{\alpha}{\left(2 s_{1}-s_{2}\right)} \sin M-\frac{1}{8} \frac{a_{1}^{2}}{a_{3}} \frac{\alpha^{2}}{\left(2 s_{1}-s_{2}\right)^{2}} \sin 2 M
\end{aligned}
$$




$$
\begin{aligned}
& -\left\{\left(\frac{1}{2} \frac{a_{1}^{2}}{a_{2}^{\frac{1}{2}}}-4 a_{1} a_{2}^{\frac{1}{2}}\right) \sin M-\frac{1}{24} \frac{a_{1}^{3}}{a_{2}^{3}} \sin 3 M\right\} \frac{a^{3}}{\left(2 s_{1}-s_{2}\right)^{3}} \\
& +\left\{\left(\frac{1}{4} \frac{a_{1}^{3}}{a_{2}}-a_{1}^{2}\right) \sin 2 M-\frac{1}{64} \frac{a_{1}^{4}}{a_{2}^{2}} \sin 4 M\right\} \frac{a^{4}}{\left(2 s_{1}-s_{2}\right)^{4}}
\end{aligned}
$$

where $\beta_{1}, \beta_{2}$, and $V$ are given by equations (13) and (14), and $\epsilon_{1}, \epsilon_{2}, a_{1}$, and $a_{2}$ are arbitrary constants depending or the circumstances of projection.

The solution is thus given in terms of infinite series, most of the terms of which certainly contain $\left(2 s_{1}-s_{2}\right)$ in the denominator. The series evidently diverge when $2 s_{1}=s_{2}$, but when $\left(2 s_{1}-s_{2}\right)$ is not small and the arbitrary constants $a_{1}$ and $a_{2}$ are small the series converge rapidly.

The following particular case illustrates the form of the numerical results.

\section{Assuming}

$$
s_{1}=1, s_{2}=1 \cdot 75, \alpha=0 \cdot 1, a_{1}=0 \cdot 5, a_{2}=0 \cdot 25, \epsilon_{1}=\epsilon_{2}=0
$$

we obtain

$$
\begin{aligned}
& h=0 \cdot 93534, M=-0 \cdot 2716 t \\
& \beta_{1}=3 \cdot 687776 M^{*}, \beta_{2}=6 \cdot 375552 M^{*} \\
p_{1}= & 3 \cdot 687776 . M+9 \cdot 626 \sin M+0 \cdot 779 \sin 2 M+0 \cdot 153 \sin 3 M \\
p_{2}= & 6 \cdot 375552 M+12 \cdot 376 \sin M-1 \cdot 329 \sin 2 M+0 \cdot 153 \sin 3 M \\
q_{1}= & 0 \cdot 5104-0 \cdot 184 \cos M+0 \cdot 0032 \cos 2 M \\
q_{2}= & 0 \cdot 2448+0 \cdot 092 \cos M-0 \cdot 0016 \cos 2 M .
\end{aligned}
$$

The original conrdinates $\phi_{1}$, and $\phi_{2}$, are then obtained by the use of equations (1). The orbit for this case is shown in figure 1, for values of $M$ between $0^{\circ}$ and $600^{\circ}$.

- As these values are required with greater accuracy further terms were obtained in the expressions for $\beta_{1}$ and $\beta_{2}$. 


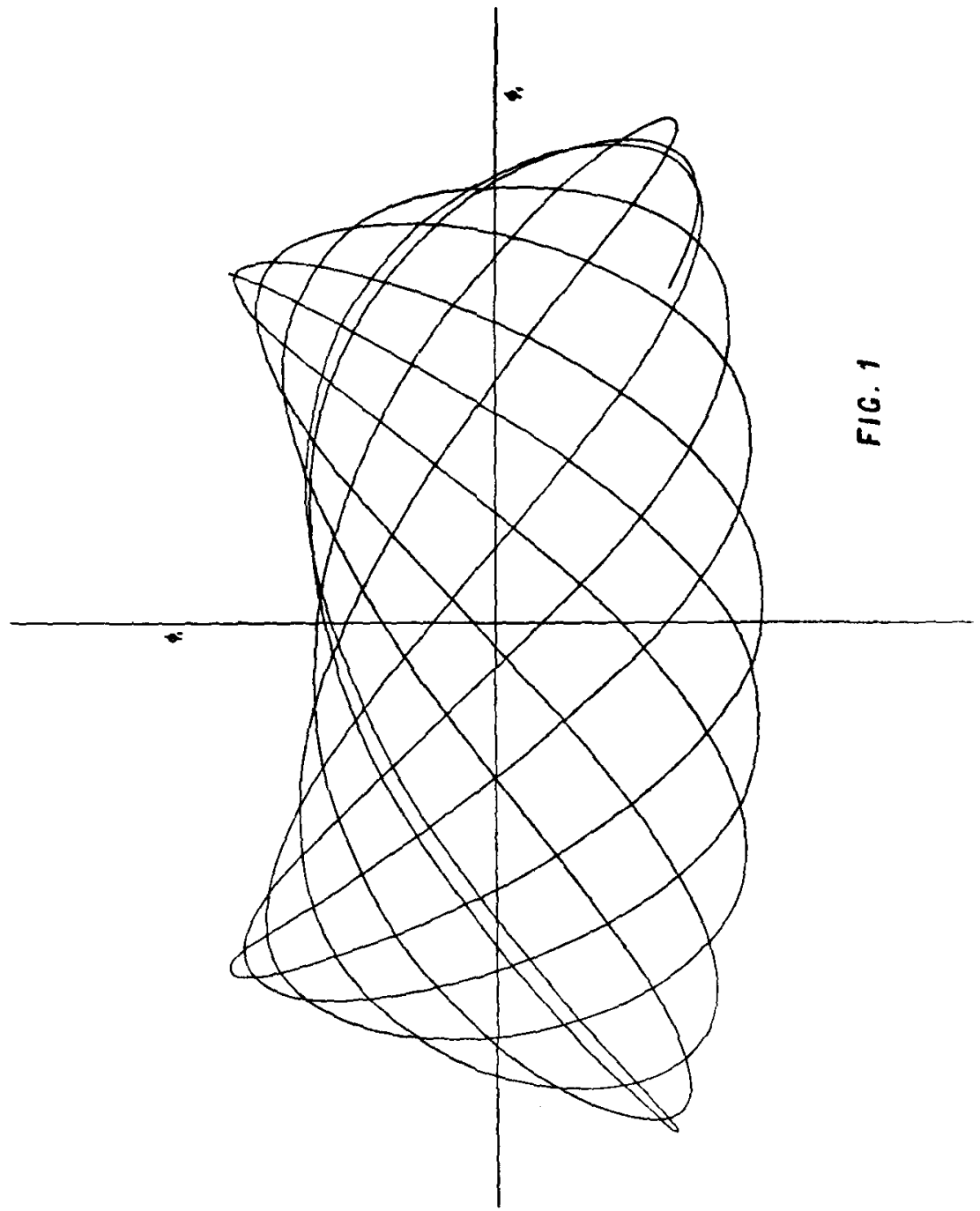

5. Elliptic Functions solution.

It is possible, however, to present the solution in a closed form by means of elliptic functions. 
From the two integrals given by equations (5) and (6), together with the second of equations (3), we have

where

$$
\begin{aligned}
\dot{q}_{2}^{2} & =\alpha^{2} q_{3}^{2} q_{2}\left\{1-\cos ^{2}\left(2 p_{1}-p_{2}\right)\right\} \\
& =\alpha^{2} q_{1}^{2} q_{2}-\left(h-s_{1} q_{1}-s_{2} q_{2}\right)^{2} \\
& =\alpha^{2}\left(c-2 q_{2}\right)^{2} q_{2}-\left\{\left(h-s_{1} c\right)+q_{2}\left(2 s_{1}-s_{2}\right)\right\}^{2} \\
& =A+B q_{2}+C q_{2}^{2}+D q_{2}^{3}, \\
& A=-\left(h-s_{1} c\right)^{2} \\
B & =\alpha^{2} c^{2}-2 h\left(2 s_{1}-s_{2}\right)+2 s_{1}\left(2 s_{1}-s_{2}\right) c, \\
C & =-\left\{\left(2 s_{1}-s_{2}\right)^{2}+4 \alpha^{2} c\right\}, \\
D & =4 \alpha^{2} .
\end{aligned}
$$

We lose no generality by replacing $c$ and $s_{1}$ by unity and it is further convenient to write

$$
1-h=g \text { and } 2 s_{1}-s_{2}=s \text {. }
$$

Then

$$
\dot{q}_{2}^{2}=4 \alpha^{2} q_{2}^{3}-\left(4 \alpha^{2}+s^{2}\right) q_{2}^{2}+\left(\alpha^{2}+2 s g\right) q_{2}-g^{2}
$$

The problem may therefore be solved in terms of elliptic functions, using equations (3) and (6) to give corresponding values of $p_{1}, p_{2}$ and $q_{1}$.

6. As an illustration of the methods adopted, we take the particular case which has already been solved by means of the series solution, in $\S 4$.

We have $s_{2}=1 \cdot 75, \alpha=0 \cdot 1, g=1-h=0 \cdot 06466, s=0 \cdot 25$; giving $A=-0.004181, B=0 \cdot 0423, C=-0 \cdot 1025, D=0 \cdot 04$;

$$
\therefore q_{2}^{2}=0 \cdot 04\left[q_{2}^{3}-2 \cdot 562 q_{2}^{2}+1 \cdot 0582 q_{2}-0 \cdot 10452\right] \text {. }
$$

The cubic in square brackets has three real positive roots, viz. :-

$$
\lambda=0 \cdot 15019, \mu=0 \cdot 3350, \nu=2 \cdot 077 .
$$

To obtain the Jacobian elliptic functions we use the transformation

$$
q_{2}=\frac{a k z+b}{k z+1}
$$

the appropriate correspondence being

$$
\left\{\begin{array}{c}
\infty, \lambda, \mu, \quad v \\
-\frac{1}{k},-1,+1,+\frac{1}{k}
\end{array}\right\} .
$$

Then

$$
\nu=\frac{a+b}{2}, \mu=\frac{a k+b}{k+1}, \lambda=\frac{b-a k}{1-k} \text {. }
$$


Eliminating $k$ we obtain

$$
a b=(\mu \nu+\nu \lambda+\lambda \mu)-2 \lambda \mu=0 \cdot 9576 ;
$$

$a$ and $b$ are therefore the roots of the quadratic equation

$$
\xi^{2}-2 v \xi+0 \cdot 9576=0
$$

whence

$$
a=3 \cdot 910, b=0 \cdot 2449 \text {, and } k=0 \cdot 02520 \text {. }
$$

The differential equation for $z$ is then

$$
\begin{aligned}
& \quad \frac{k^{2}(a-b)^{2}}{0 \cdot 04}\left(\frac{d z}{d t}\right)^{2}=(b-\lambda)(\mu-b)(\nu-b)\left(1-z^{2}\right)\left(1-k^{2} z^{2}\right) . \\
& \text { Put } u=-\frac{\sqrt{0 \cdot 04(b-\lambda)(\mu-b)(\nu-b)}}{k(a-b)} \cdot t=-0.2708 t ;
\end{aligned}
$$

$u$ therefore corresponds closely to $M$ of the series solution (see $\S 4$ ).

We have then

and

$$
z=\operatorname{sn}(u, k)
$$

$$
q_{2}=0 \cdot 2449+\frac{0 \cdot 09236 \operatorname{sn} u}{1+0 \cdot 02520 \operatorname{sn} u},
$$

$q_{1}$ being given by $q_{1}=1-2 q_{2}$.

$$
\text { Further, } \dot{p}_{1}=-\frac{\partial H}{\partial q_{1}}=\frac{h-s_{2} q_{2}}{q_{1}}
$$

and

$$
\dot{p}_{2}=-\frac{\partial H}{\partial q_{2}}=-\frac{1}{2 q_{2}}\left(h-s_{1} q_{1}+s_{2} q_{2}\right)
$$

values of $p_{1}$ and $p_{2}$ can therefore be obtained, by using the known values of $q_{1}$ and $q_{2}$, and integrating these equations.

7. The method will also give a solution when the series solution is no longer of use. We have already shown that when $s=0$, i.e. $s_{2}=2$, the series for $q_{1}, q_{2}, p_{1}$ and $p_{2}$ are certainly divergent.

Assume now $s=0, g=0 \cdot 02, \alpha=0 \cdot 1$.

The solution then reduces to

$$
\begin{aligned}
q_{2}= & \frac{0 \cdot 16761353 \operatorname{sn} u+0 \cdot 20892106}{0 \cdot 16058204 \operatorname{sn} u+1}, \\
q_{1}= & 1-2 q_{2}, \\
p_{1}= & 7 \cdot 35622241 u-\frac{1}{2} \tan ^{-1}[3 \cdot 76458405 \mathrm{dc} u] \\
& +[0 \cdot 01015432 \sin 2 v-0 \cdot 00010177 \sin 4 v \\
& \quad+0 \cdot 00000137 \sin 6 v-0 \cdot 00000002 \sin 8 v],
\end{aligned}
$$




$$
\begin{aligned}
p_{2} & =14 \cdot 71296994 u+\frac{1}{2} \tan ^{-1}[0 \cdot 75942794 \mathrm{dc} u] \\
& +\left[\frac{v}{2}-\frac{1}{2} \tan ^{-1}\{0 \cdot 60375960 \tan v\}\right]-0 \cdot 00000507 \sin 2 v,
\end{aligned}
$$

where $v=\frac{\pi}{2 K} u$ and $u=-0 \cdot 130917125 t$.

The orbit, for values of $v$ between $0^{\circ}$ and $360^{\circ}$, is shown in figure 2.

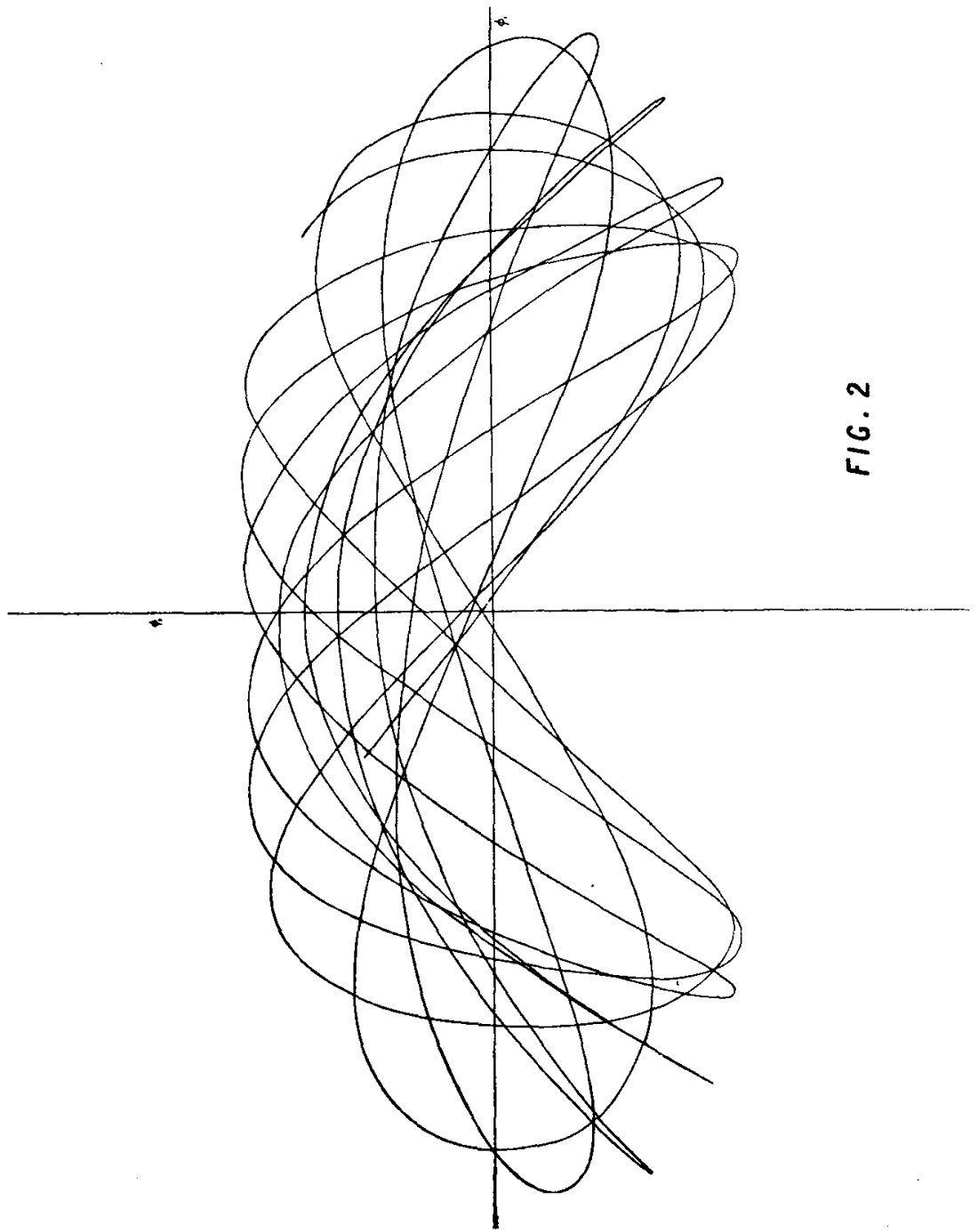




\section{Conditions for a Real Solution.}

From the first two of equations (1) it is apparent that for real values of the coordinates $\phi_{1}$ and $\phi_{2}$, the two coordinates $q_{1}$ and $q_{2}$ must be both positive, and therefore, from equation (6), neither $q_{1}$ nor $2 q_{2}$ must exceed $c$ (which we have taken, arbitrarily, to be unity).

The satisfying of this condition depends upon the roots of the cubic on the right hand side of equation (16), viz. :-

$$
4 \alpha^{2} q_{2}^{3}-\left(4 \alpha^{2}+s^{2}\right) q_{2}^{2}+\left(\alpha^{2}+2 s g\right) q_{2}-g^{2} .
$$

From the form of the coefficients it follows that the roots of this cubic must be either all real and positive or else one real and two imaginary. When the roots are all real, as in the particular cases already discussed in $\$ 6$ and 7 , a real solution is obtained.

As a particular example of a case in which two roots are imaginary, assume

$$
s=0, g=0 \cdot 06466, \alpha=0 \cdot 1
$$

giving $\quad \dot{q}_{2}^{2}=0 \cdot 04\left(q_{2}-0 \cdot 8506\right)\left(q_{2}^{2}-0 \cdot 14945 q_{2}+0 \cdot 12289\right)$

we deduce

$$
q_{2}=\frac{1 \cdot 6986+0 \cdot 002487 \mathrm{cn} u}{1+\operatorname{cn} u} .
$$

Thus $q_{2}>\frac{1}{2}$ and $\therefore q_{1}<0$ and the solution is imaginary. It thus appears that a condition for a real solution is that the roots of the cubic on the right hand side of equation (16) should be all real and therefore all positive. The transition occurs when the discriminant of the cubic is zero. Denoting this discriminant by $\Delta$ we obtain

$$
\Delta=\alpha^{2}(s-2 g)^{2}\left\{8 \alpha^{4}+\alpha^{2}\left(s^{2}+36 s g-108 g^{2}\right)+4 s^{3} g\right\}
$$

The locus represented by $\Delta=0$, taking $s$ and $g$ as the two variables, consists of two curved branches and the repeated straight line $s=2 g$ (referred to in the sequence as "the double line"), which touches each curved branch. The locus, for the value $\alpha=0 \cdot 1$, is shown in figure 3.

For real roots we must have $\Delta>0$, i.e., the point $(s, g)$ must lie between the curved branches. 
To discuss the conditions more fully, we consider the differential equation (16), satisfied by $q_{2}$, and in it take $u= \pm \alpha t$ as the independent variable and $\left(q_{2}-\frac{4 \alpha^{2}+s^{2}}{12 \alpha^{2}}\right)$ as the dependent variable,

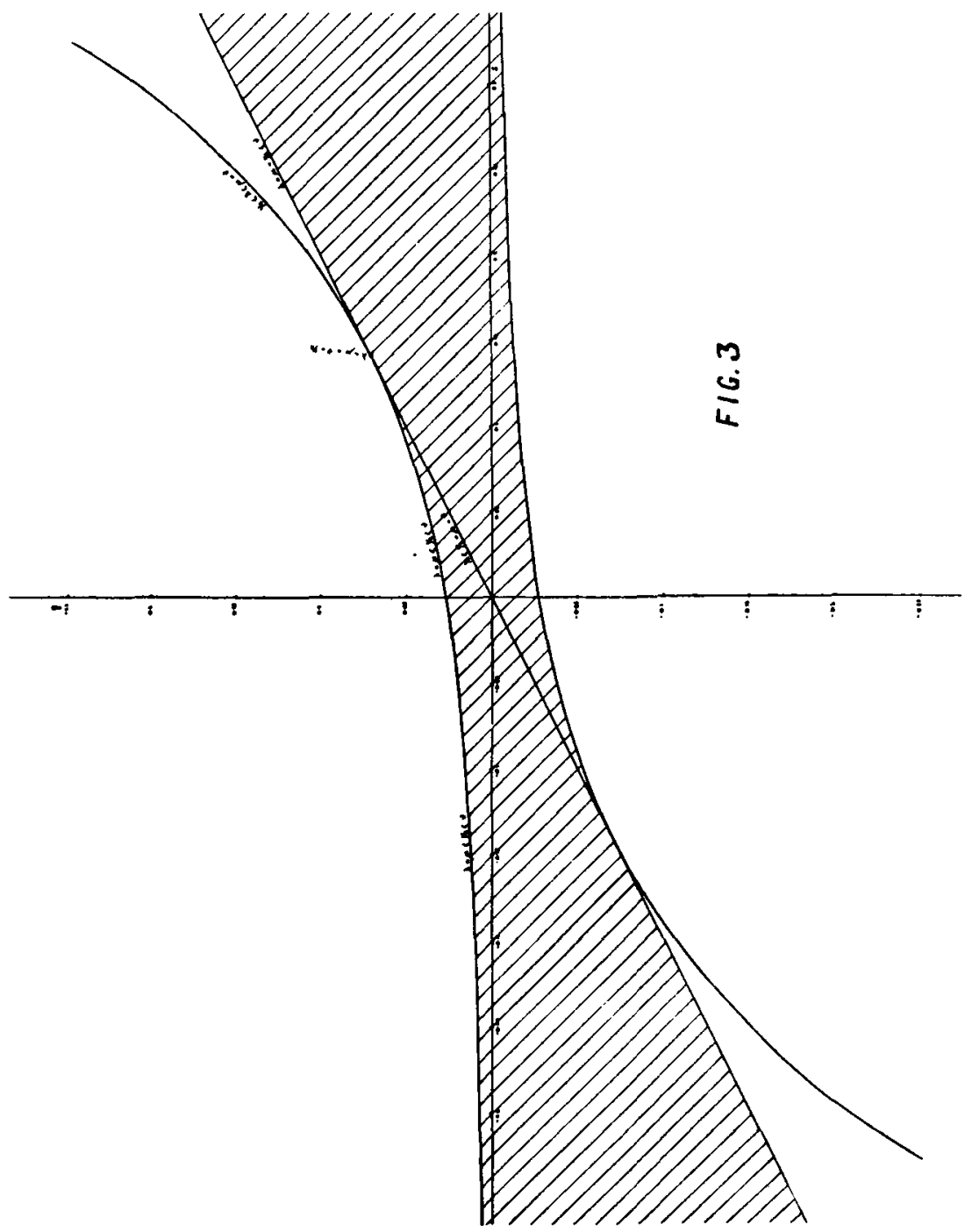


denoting it by $\wp(u)$. This gives the ordinary equation for the Weierstrassian elliptic function, viz.:-

$$
\left\{\frac{d \wp(u)}{d u}\right\}^{2}=4\{\wp(u)\}^{3}-g_{9} \wp(u)-g_{3}
$$

$g_{2}$ and $g_{3}$ being constants depending on $\alpha, s$ and $g$.

Then

$$
\begin{aligned}
& q_{2}=\wp(u)+\frac{4 \alpha^{2}+s^{2}}{12 \alpha^{2}}, \\
& q_{1}=\frac{4 \alpha^{2}-2 s^{2}}{12 \alpha^{2}}-2 \wp(u) .
\end{aligned}
$$

For a real solution $q_{1}, q_{2}$ and $\left\{\frac{d \varphi(u)}{d u}\right\}^{2}$ must all be positive and this is only possible on the oval branch of the curve

$$
y^{2}=4 x^{3}-g_{2} x+g_{3}
$$

when the three roots of the cubic are real.

If $e_{1}, e_{2}, e_{3}$ be the roots of the cubic on the right hand side of equation (18), and if $e_{1}>e_{2}>e_{3}$ we must have $e_{2} \geqq \wp(u) \geqq e_{3}$.

Suppose $\lambda, \mu, \nu$ to be the roots of the cubic on the right hand side of equation (16), where $\lambda<\mu<v$, then the conditions for a real periodic solution in $q_{2}$ are

and

$$
\frac{4 \alpha^{2}+s^{2}}{12 \alpha^{2}}+e_{3}>0, \text { i.e. } \lambda>0
$$

$$
\frac{4 \alpha^{2}+s^{2}}{12 \alpha^{2}}-2 e_{2}>0, \text { i.e. } 1-2 \mu>0 .
$$

The conditions are therefore:-

(i) all the roots of the cubic in $q_{2}$ must be real and positive,

(ii) $\mu$, the middle root, must be less than $\frac{1}{2}$.

On substituting $q_{2}=\frac{1}{2}$ in the right hand side of equation (16), we obtain $-\frac{1}{4}(s-2 g)^{2}$, so that either two roots or no roots are less than $\frac{1}{2}$ (i.e. the greatest root is al ways greater than $\frac{1}{2}$ ), except when $s=2 g$, in which case two roots equal $\frac{1}{2}$.

When $s=2 g$, the third root is $\frac{s^{2}}{4 \alpha^{2}}$; it is therefore the smallest root if $s^{2}<2 \alpha^{2}$ and the greatest root if $s^{2}>2 \alpha^{2}$; when $s^{2}=2 \alpha^{2}$ we obtain one or other of the points at which the double line, $s=2 g$, touches the curved branches, and here all the roots are equal and equal to $\frac{1}{2}$. 
9. To investigate the behaviour on the curved branches of the curve $\Delta=0$, we equate the discriminant to zero and solve for $g$, giving

$$
g=\frac{s^{3}+9 s \alpha^{2}+R^{3}}{54 \alpha^{2}} \text { where } R= \pm \sqrt{s^{2}+6 \alpha^{2}}
$$

The cubic for $q_{3}$, viz. :-

$$
4 \alpha^{2} q_{2}^{3}-\left(4 \alpha^{2}+s^{2}\right) q_{2}^{2}+\left(\alpha^{2}+2 s g\right) q_{2}-g=0,
$$

has two equal roots and therefore the derivate also vanishes when $g$ has the values given by equation (19). We must therefore have either

$$
q_{2}=\frac{9 \alpha^{2}+2 s^{2}-R s}{18 \alpha^{2}} \text { or } q_{2}=\frac{3 \alpha^{2}+s^{2}+R s}{18 \alpha^{2}},
$$

the second of these being the double root of the cubic. The remaining root is found to be

$$
q_{2}=\frac{24 \alpha^{2}+5 s^{2}-4 R s}{36 \alpha^{2}} .
$$

These roots reduce to

$$
a=\frac{(R+s)^{2}}{36 \alpha^{2}}, b=\frac{(2 R-s)^{2}}{36 \alpha^{2}},
$$

$a$ being the repeated root.

We have now to investigate under what circumstances $a$ is the greater root. If $a>b$ the middle root will be greater than $\frac{1}{2}$, and no real solution will be obtained, since the greatest root is always greater than $\frac{1}{2}$ (except at points on the double line).

Now $\quad a>b$ if $(R+s)^{2}>(2 R-s)^{2}$ i.e. if $2 R s>R^{2}$.

This cannot be the case if $R$ and $s$ have different signs. If they have the same sign, suppose they are both positive and the condition reduces to $s^{2}>2 \alpha^{2}$.

We have, then,

(i) $g>0, s>0, s^{2}>2 \alpha^{2}$; all the roots are $>\frac{1}{2}$,

(ii) $g>0, s>0, s^{2}<2 \alpha^{2}$; two roots are equal and $<\frac{1}{2}$,

(iii) $g>0, s<0$, two roots are equal and $<\frac{1}{2}$.

The areas towards infinity, between the double line and the curved branches, must therefore be excluded from the permissible area (see figure 3). (The permissible area is shaded in the figure.) 
The particular forms of the orbits on the boundaries of the permissible area (including the double line) are discussed below in $\$ 10$.

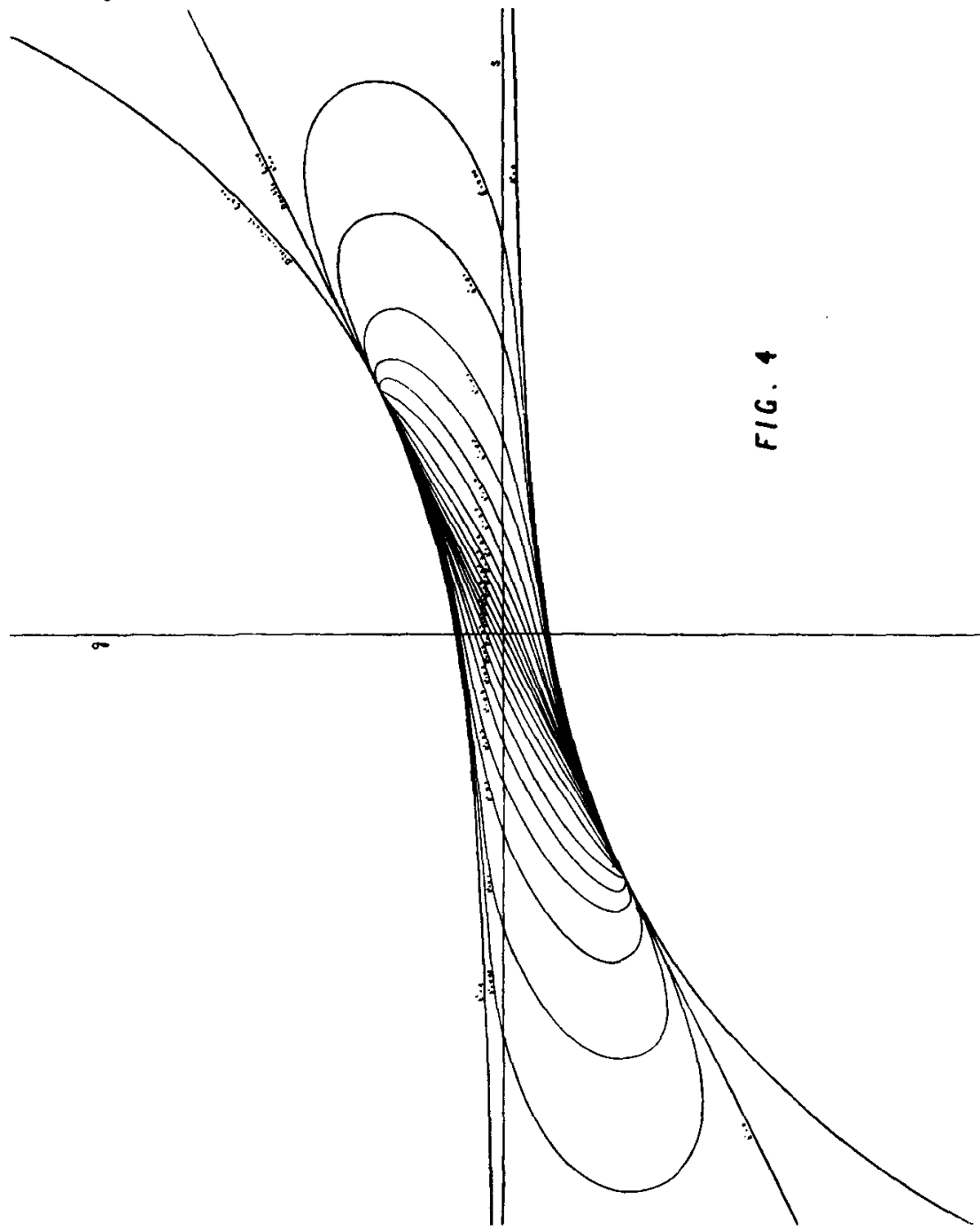

In figure 4 are sketched curves showing the values of 8 and $g$ for different constant values of $k^{2}$, in the permissible region, where 
51

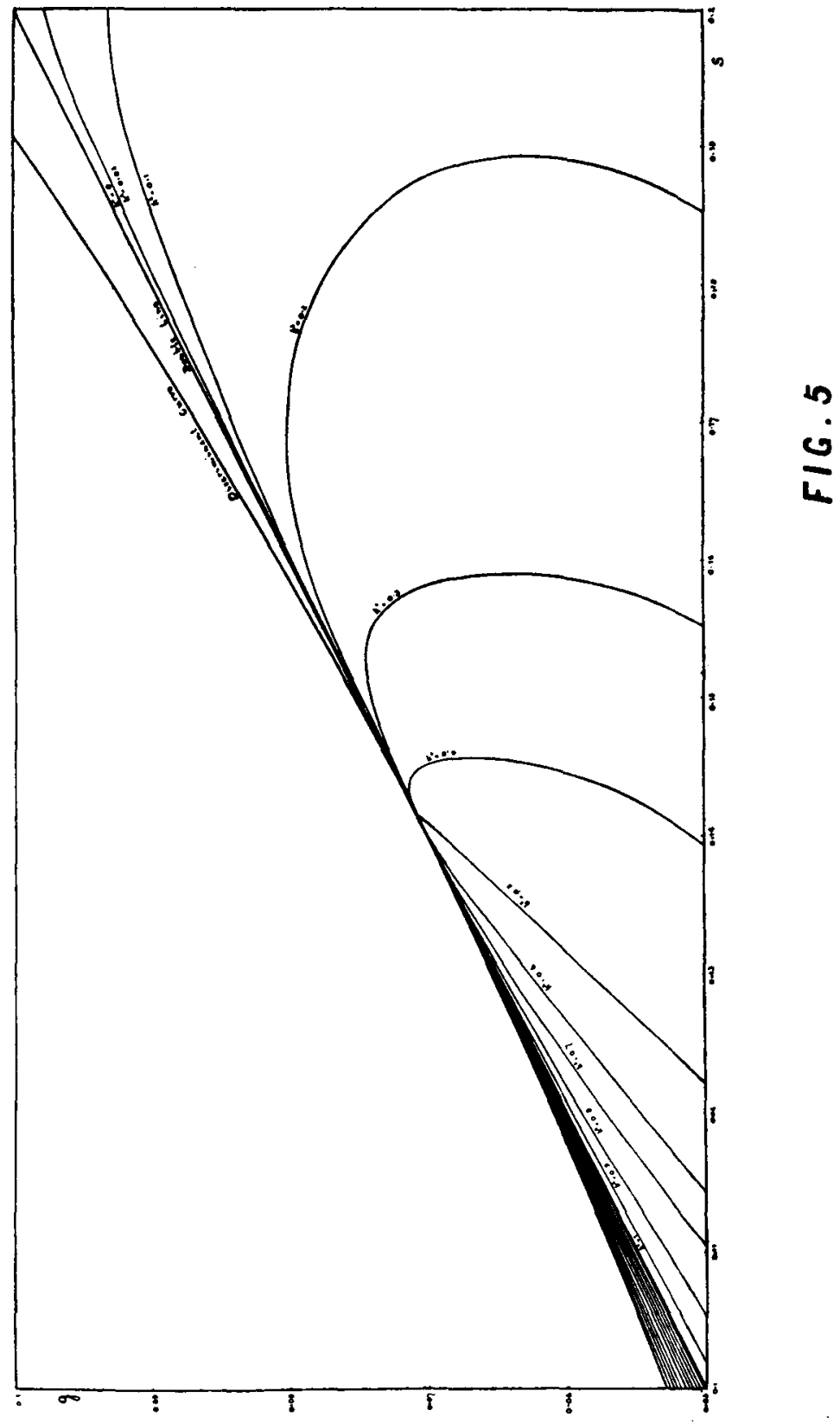


$k$ is the modulus of the Weierstrassian elliptic functions occurring in the solutions for $q_{1}$ and $q_{2}$, and is thus related to the period of the vibration. Near the points at which the double line touches the curved branches (i.e. where all the roots are equal) we get the somewhat curious result, that, if we approach these points along any straight line, making a finite angle with the double line, the value of $k^{3}$ tends to $\frac{1}{2}$. The curves for constant values of $k^{2}$, in the immediate neigbourhood of these points are shown on a larger scale in figure 5 .

\section{Limiting Forms of the Solution.}

We divide the discussion of particular limiting forms of the solution into four cases.

Case 1.-Points on the Double Line $s=2 g$.

$1^{\circ}$ Points betrceen the points of contact with the curved branches of the discriminant.

In this case $b^{2}<2 a^{2}$ and

Putting

$$
\dot{q}_{2}^{2}=\left(q_{2}-\frac{1}{2}\right)^{2}\left(4 \alpha^{2} q_{2}-8\right) .
$$

$$
\frac{1}{2}-q_{2}=\frac{1}{x^{2}} \quad \therefore 4 \dot{x^{2}}=\left(2 \alpha^{2}-s^{2}\right) x^{2}-4 \alpha^{2}
$$

we obtain

$$
\begin{aligned}
& q_{2}=\frac{s^{2}}{4 \alpha^{2}}+\frac{1}{2}\left(1-\frac{s^{2}}{2 \alpha^{2}}\right) \tanh ^{2}\left\{\frac{\sqrt{2 \alpha^{2}-s^{2}}}{2} \cdot t\right\} \\
& q_{1}=\left(1-\frac{s^{2}}{2 \alpha^{2}}\right) \operatorname{sech}^{2}\left\{\frac{\sqrt{2 \alpha^{2}-s^{2}}}{2} \cdot t\right\} \\
& p_{1}=-\frac{s_{2}}{2} \cdot t+\text { constant, } \\
& p_{2}=\text { constant }-s_{2} t+\tan ^{-1}\left\{\frac{\sqrt{2 \alpha^{2}-s^{2}}}{s} \tanh \left(\frac{\sqrt{2 \alpha^{2}-s^{2}}}{2} \cdot t\right)\right\} .
\end{aligned}
$$

The whole of the energy is thus finally absorbed by $\phi_{2}$.

The form of the orbit for the values:- $g=0.02, a=0 \cdot 1$, $s=0.04$, is shown in figure 6 . It is noteworthy that, when $\phi_{1}=0, \phi_{2}$ has always the same value when $t$ is finite. 


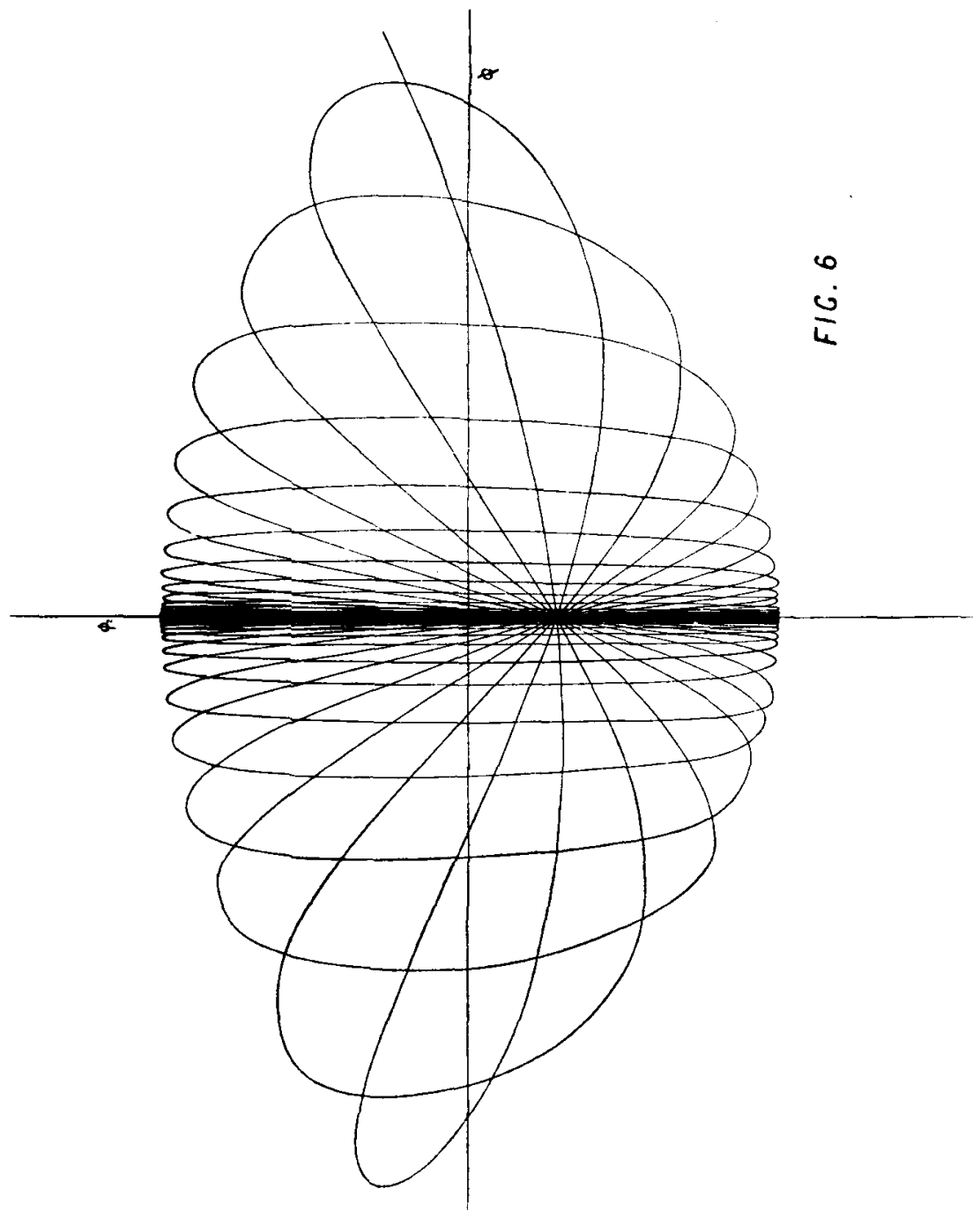

$2^{\circ}$ Points outside the points of contact urith the curved branches of the discriminant.

In this case $s^{2}>2 \alpha^{2}$ and we obtain as the only real solution

$$
\begin{aligned}
& q_{2}=\frac{1}{2}, \quad q_{1}=0 \text { permanently, } \\
& p_{2}=-s_{2} t+\text { constant, } p_{1} \text { is indeterminate. }
\end{aligned}
$$


This gives merely a simple harmonic motion in $\phi_{2}$, of period $\frac{2 \pi}{s_{2}}$.

CASE 2.-The point of contact of the Curved Branches of the Discriminant with the Double Line.

In this case $g=\frac{s}{2}=\frac{\alpha}{\sqrt{2}}$,

giving $q_{2}=\frac{1}{2}, \quad q_{1}=0, \quad p_{2}=-s_{2} t+$ constant.

We have therefore, as the complete solution, the same simple harmonic vibration as in Case $1,2^{\circ}$.

CASE 3. -The origin, $g=s=0$.

We obtain, either $q_{2}=0, q_{1}=1$

$$
\text { or } \quad q_{2}=\frac{1}{2}, q_{1}=0
$$

and $p_{1}=-t+$ constant, $p_{2}=-2 t+$ constant.

We have, therefore, either a simple harmonic vibration in $\phi_{1}$ of period $2 \pi$, or a simple harmonic vibration in $\phi_{2}$ of period $\pi$.

CAsE 4.-The part of the Curved Branches of the Discriminant which is a Boundary of the Permissible Region.

We have

$$
\dot{q}_{2}^{2}=4 \alpha^{2}\left(q_{2}-\lambda\right)^{2}\left(q_{2}-v\right) \text { where } v>\frac{1}{2}>\lambda>0 .
$$

The only valid part of the solution is given by

$$
\begin{aligned}
& q_{2}=\lambda, q_{1}=1-2 \lambda, \\
& p_{1}=\left\{-1+\frac{s}{2}+\frac{8-2 g}{2(1-2 \lambda)}\right\} t+\text { constant } \\
& p_{2}=\left\{-2+\frac{s}{2}+\frac{g}{2 \lambda}\right\} t+\text { constant } .
\end{aligned}
$$

\section{General Solution.}

To obtain the solution in the general case, we assume the cubic on the right hand side of equation (16) to have roots $\lambda, \mu, \nu$, where $\lambda<\mu<\nu$, and suppose the conditions for a real solution to be satisfied. 
Effecting on $q_{2}$ a linear transformation, given by

$$
q_{2}=\frac{m k z+l}{k z+1}
$$

where $\quad v=\frac{l+m}{2}, \mu=\frac{l+m k}{1+k}, \lambda=\frac{l-m k}{1-k}$,

and $0<k m<l<m$ and $0<\lambda<l<\mu<v<m$, equation (16) reduces to

$$
\dot{z}^{2}=\frac{4 \alpha^{2}(l-\lambda)(l-\mu)(l-v)}{k^{2}(m-l)^{2}}\left(1-z^{2}\right)\left(1-k^{2} z^{2}\right) .
$$

If we put $\dot{u}^{2}=\frac{4 \alpha^{2}(l-\lambda)(l-\mu)}{k^{2}(m-l)^{2}} \frac{(l-v)}{\text {, we obtain }}$

and therefore

$$
z=\operatorname{sn}(u, k)
$$

$$
q_{2}=\frac{m k \sin u+l}{1+k \sin u}
$$

$k, m, l$, being determined by equations $(21)$.

Then

$$
q_{1}=1-2 q_{2}=\frac{1-2 l-k(2 m-1) \operatorname{sn} u}{1+k \operatorname{sn} u}
$$

Also

Taking

$$
\begin{aligned}
\dot{p}_{1} & =-\frac{\partial H}{\partial q_{1}}=\left[-s_{1}+\frac{h-s_{1}}{q_{1}-s_{2} q_{2}} q_{1}\right] \\
& =-\frac{2-s}{2}-\frac{8-2 g}{2 q_{1}} .
\end{aligned}
$$

we obtain

$$
\frac{d u}{d t}=-N=-\frac{2 \alpha}{k(m-l)} \sqrt{(l-\lambda)(l-\mu)(l-\nu)},
$$

$$
\begin{gathered}
\frac{d p_{1}}{d u}=\frac{1}{2 N}\left\{2-s+\frac{s-2 g}{2 q_{1}}\right\} ; \\
\therefore p_{1}-\frac{2-8}{2 N} \cdot u=\frac{s-2 g}{N} \int \frac{1+k \operatorname{sn} u}{1-2 l-k(2 m-1) \operatorname{sn} u} \cdot d u \\
=\frac{(s-2 g)}{2 N(1-2 l)} \cdot u+\frac{(s-2 g)(1+\operatorname{sn} a)}{2 N(1-2 l)} \int \frac{k \operatorname{sn} u(1+k \operatorname{sn} u \operatorname{sn} a)}{1-k^{2} \operatorname{sn}^{2} u \cdot \operatorname{sn}^{2} a} \cdot d u \\
\text { where sn } a=\frac{2 m-1}{1-2 l} .
\end{gathered}
$$


This reduces to

$$
\begin{aligned}
& p_{1}=\left\{\frac{2-s}{2 N}+\frac{s-2 g}{2 N(1-2 l)}\right\} u \\
& +\frac{(s-2 g)(1+\operatorname{sn} a)}{2 N(1-2 l) C \operatorname{dn} a}\left[\tan ^{-1}\left\{\frac{\operatorname{dc} u \operatorname{dn} a}{k C}\right\}+i \cdot u \frac{\Theta^{\prime}(a)}{\Theta(a)}+\frac{i}{2} \cdot \log _{e} \frac{\Theta(u-a)}{\Theta(u+a)}\right] \\
& \text { where cn } a=-i C ; \\
& \therefore p_{1}=\left\{\frac{2-s}{2 N}+\frac{s-2 g}{2 N(1-2 l)}\right\} u \\
& \pm \frac{1}{2}\left[\tan ^{-1}\left\{\frac{\operatorname{dc} u \operatorname{dn} a}{k} \frac{d}{C}\right\}+i u \frac{\Theta^{\prime}(a)}{\Theta(a)}+\frac{i}{2} \cdot \log _{e} \frac{\Theta(u-a)}{\Theta(u+a)}\right],
\end{aligned}
$$

the sign outside the square bracket being the same as that of $(s-2 g)$.

$$
\begin{aligned}
& \text { Similarly } \dot{p}_{2}=-\frac{\partial H}{\partial q_{2}}=-\frac{4-s}{2}+\frac{g}{2 q_{2}} ; \\
& \therefore \frac{d p_{2}}{d u}=\frac{4-8}{2 N}-\frac{g}{2 N} \cdot \frac{1+k \operatorname{sn} u}{l+k m \operatorname{sn} u} \\
& \therefore p_{2}=\frac{u}{2 N}\left(4-8-\frac{g}{l}\right)+\frac{g\left(\operatorname{sn} a^{\prime}-1\right)}{2 l N} \int \frac{k \operatorname{sn} u\left(1-k \operatorname{sn} u \operatorname{sn} a^{\prime}\right)}{1-k^{2} \operatorname{sn}^{2} u \cdot \operatorname{sn}^{2} a^{\prime}} \cdot d u \\
& \text { where } \operatorname{sn} a^{\prime}=\frac{m}{l} .
\end{aligned}
$$

Putting cn $a^{\prime}=-i C,^{\prime}$ this reduces to

$$
\begin{aligned}
p_{2} & =\left\{4-s-\frac{g}{l}\right\} \frac{u}{2 N} \\
& \pm \frac{1}{2}\left[\tan ^{-1}\left\{\frac{\operatorname{dc} u \operatorname{dn} a^{\prime}}{k} \frac{C^{\prime}}{C^{\prime}}+i u \frac{\Theta^{\prime}\left(a^{\prime}\right)}{\Theta\left(a^{\prime}\right)}+\frac{i}{2} \cdot \log _{e} \frac{\Theta\left(u-a^{\prime}\right)}{\Theta\left(u+a^{\prime}\right)}\right],\right.
\end{aligned}
$$

the sign outside the square bracket being the same as that of $g$.

Some modifications are necessary when $g=0$.

12. The solution of the particular problem is thus complete, a solution being obtained in every case in which a real solution exists. We have not yet been able to commence the most 
important line of research suggested by the work, namely, the investigation of the cause of divergence of the series solution; that it is a case of failure to represent the state of affiairs, and not an indication of any discontinuity in the system, is shown by the existence of the elliptic function solution. The chief feature of the latter is the presence of the double line with its peculiar properties. So far as we know, attention has not previously been directed to the fact, that it is not for the perfect octave $s=0$, that the greatest deviation from the general type of solution occurs, but along the double line, $s=2 g$. That this line is double, suggests that, when other less important and suitably chosen terms are introduced into the fundamental expression for $H$, equation (2), it may open out into a region in which no orbit is possible.

We have to thank Professor Whittaker, not only for introducing us to the problem and for the interest and help with which he has followed our work, but also for the opportunity of co-operation which the Mathematical Institute has afforded us. 